\title{
Nonpharmacological Strategies to Prevent Contrast-Induced Acute Kidney Injury
}

\author{
Paweena Susantitaphong ${ }^{1,2}$ and Somchai Eiam-Ong ${ }^{1}$ \\ ${ }^{1}$ Division of Nephrology, Department of Medicine, Faculty of Medicine, Chulalongkorn University, \\ King Chulalongkorn Memorial Hospital, Bangkok 10330, Thailand \\ ${ }^{2}$ Extracorporeal Multiorgan Support Dialysis Center, Division of Nephrology, Department of Medicine, \\ King Chulalongkorn Memorial Hospital, Faculty of Medicine, Chulalongkorn University, Bangkok 10330, Thailand
}

Correspondence should be addressed to Somchai Eiam-Ong; somchai80754@yahoo.com

Received 30 December 2013; Revised 19 February 2014; Accepted 20 February 2014; Published 26 March 2014

Academic Editor: Michele Andreucci

Copyright (C) 2014 P. Susantitaphong and S. Eiam-Ong. This is an open access article distributed under the Creative Commons Attribution License, which permits unrestricted use, distribution, and reproduction in any medium, provided the original work is properly cited.

Contrast-induced AKI (CI-AKI) has been one of the leading causes for hospital-acquired AKI and is associated with independent risk for adverse clinical outcomes including morbidity and mortality. The aim of this review is to provide a brief summary of the studies that focus on nonpharmacological strategies to prevent CI-AKI, including routine identification of at-risk patients, use of appropriate hydration regimens, withdrawal of nephrotoxic drugs, selection of low-osmolar contrast media or isoosmolar contrast media, and using the minimum volume of contrast media as possible. There is no need to schedule dialysis in relation to injection of contrast media or injection of contrast agent in relation to dialysis program. Hemodialysis cannot protect the poorly functioning kidney against CI-AKI.

\section{Introduction}

The definition of acute kidney injury (AKI) is reclassified according to the Kidney Disease Improving Global Outcomes (KDIGO) staging system. Using the KDIGO definition, 1 in 5 adults and 1 in 3 children worldwide experience AKI during a hospital episode of care [1]. Contrast-induced AKI (CI-AKI) is defined as a $\geq 0.5 \mathrm{mg} / \mathrm{dL}$ rise or a $25 \%$ increase in serum creatinine, assessed within 48-72 hours after administration of contrast media. CI-AKI is one of the most common causes of hospital-acquired AKI. The incidence varies from less than $2 \%$ in general population up to $50 \%$ in patients with advanced kidney disease [2]. A recent meta-analysis regarding incidence of CI-AKI after contrast-enhanced computed tomography (CT) showed that the pooled incidence of CIAKI was 6.4\% (95\% CI 5.0-8.1). The risk of renal replacement therapy requirement after CI-AKI was low $(0.06 \% 95 \%$ CI 0.01-0.4). The decline in renal function persisted in $1.1 \%$ of patients (95\% CI $0.6-2.1 \%)$. Patients with chronic kidney disease (CKD) (odds ratio 2.26, $P<0.001$ ) or diabetes mellitus (odds ratio 3.10, $P<0.001$ ) were at increased risk for the development of CI-AKI [3]. CI-AKI is also associated with an increased risk of mortality, cardiovascular events, CKD, and prolonged hospitalization [4].

The number of published studies on CI-AKI has dramatically increased during the past decade. Since CI-AKI is a potentially preventable clinical condition, the more the knowledge regarding CI-AKI is understood, the greater the likelihood of reducing the risk. The aim of this review is to provide a brief review and summary of the studies that focus on nonpharmacological strategies to prevent CI-AKI.

\section{Pathophysiology of CI-AKI}

Besides direct tubular toxicity and intraluminal obstruction, renal hypoxia and concomitant release of reactive oxygen species (ROS) have also been considered as important mechanisms of renal injury in CI-AKI $[5,6]$. Hypoxia results from the imbalance of oxygen supply from renal blood flow and oxygen demand from renal tissue. Impaired oxygen supply is caused by the reduction of effective renal cortical-medullary blood flow and afferent arteriole constriction mediated 
TABLE 1: Risk factors for contrast-induced acute kidney injury.

\begin{tabular}{|c|c|}
\hline Patient-related & Procedure-related \\
\hline $\begin{array}{l}\text { (i) eGFR less than } 60 \mathrm{~mL} / \mathrm{min} / 1.73 \mathrm{~m}^{2} \text { before intra-arterial } \\
\text { administration } \\
\text { (ii) eGFR less than } 45 \mathrm{~mL} / \mathrm{min} / 1.73 \mathrm{~m}^{2} \text { before intravenous } \\
\text { administration } \\
\text { (iii) In particular, in combination with } \\
\text { diabetic nephropathy } \\
\text { dehydration } \\
\text { congestive heart failure (NYHA grade } 3-4 \text { ) and low LVEF } \\
\text { recent myocardial infarction ( }<24 \text { hours) } \\
\text { intra-aortic balloon pump } \\
\text { periprocedural hypotension } \\
\text { low hematocrit level } \\
\text { age over } 70 \text { years } \\
\text { concurrent administration of nephrotoxic drugs } \\
\text { (iv) Known or suspected acute kidney injury }\end{array}$ & $\begin{array}{l}\text { (i) Intra-arterial administration of contrast media } \\
\text { (ii) High osmolality agents } \\
\text { (iii) Large doses of contrast media } \\
\text { (iv) Multiple contrast media administrations within a few days }\end{array}$ \\
\hline
\end{tabular}

via the tubuloglomerular feedback from osmotic diuresis [7].

In addition, hyperosmotic contrast media also cause diuresis and natriuresis that stimulate the macula densa to release adenosine for the activation of adenosine A1 receptors, resulting in vasoconstriction of the afferent arteriole of the glomerulus as well as the medullary vascular bed [6]. Regarding oxygen demand, the increased reabsorption from osmotic load could accelerate metabolism and results in heightened oxygen consumption in the kidney, finally leading to the microvascular damage and intrarenal hypoxia [7].

Furthermore, renal medullary hypoxia may also produce ROS to scavenge nitric oxide (NO). Superoxide $\left(\mathrm{O}_{2}{ }^{-}\right)$, hydrogen peroxide $\left(\mathrm{H}_{2} \mathrm{O}_{2}\right)$, and hydroxyl radical $\left(\mathrm{OH}^{-}\right)$are the most common ROS [6]. Moreover, the production of these ROS could be aggravated by oxidative stress in the mitochondria [6]. Increased markers of ROS were evident in the urine and plasma of patients after cardiac catheterization, particularly in those with CKD and diabetes mellitus [8].

\section{Nonpharmacological Strategies to Prevent CI-AKI}

3.1. Evaluation of the Risk of CI-AKI and Consideration of Alternative Imaging Methods. All patients going to receive contrast media should be evaluated for the risk of CI-AKI. Prophylaxis with therapies that are supported by clinical evidence should be considered in high-risk patients. If it is possible, alternative imaging methods without contrast media in high-risk patients should be performed.

Moos et al. [9] summarized the incidence of CI-AKI and the associations between CI-AKI incidence and risk factors in patients undergoing intravenous contrast-enhanced CT with iodinated low- or isoosmolar contrast media (LOCM and IOCM, resp.). The authors reported that the overall pooled CI-AKI incidence was 4.96\% (95\% CI: 3.79-6.47) and found the significant associations between CI-AKI and those who had renal insufficiency, diabetes mellitus, and malignancy, are of age $>65$ years, and used nonsteroidal anti-inflammatory drugs (NSAIDs). Moreover, despite having a normal baseline creatinine, diabetic patients are at an increased risk of developing CI-AKI, particularly older patients and patients with high urine albumin/creatinine ratio [10].

Serum uric acid level [11], neutrophil-to-lymphocyte ratio as a systemic inflammation marker [12], pulse pressure [13], aortic stiffness represented as pulse wave velocity and augmentation index [14], and anemia [15, 16] are simple independent early predictors of CI-AKI in patients who were exposed to contrast media and are probably used for early detection that may attenuate the progression of CI-AKI.

Gurm et al. [17] also proposed the model for predicting CI-AKI including patient's conditions such as the presence of heart failure and cardiogenic shock as well as the values of laboratory setting such as hemoglobin and creatinine (area under the receiver-operating characteristic curve (AUC) for predicting CI-AKI $=0.85)$. The details of this model can be downloaded from the website https://bmc2.org/calculators/cin.

Recently, the Contrast Media Safety Committee of European Society of Urogenital Radiology (ESUR) has reported the risk factors for CI-AKI (Table 1) [18].

Electronic Warning Systems. Cho et al. [19] demonstrated the benefit of using computerized alertness program in hospitalized patients. When contrast-enhanced CT was ordered in patients with a GFR of $<60 \mathrm{~mL} / \mathrm{min} / 1.73 \mathrm{~m}^{2}$, the physician was immediately alerted by a warning message to consider prophylactic measures for CI-AKI. This electronic warning systems significantly decreased the risk of CI-AKI (3\% versus $10 \%, P=0.02$ ) [19].

3.2. Drug Review and Medication Discontinuation. Prior to contrast media exposure, the use of established nephrotoxic drugs, for example, cyclosporine A, aminoglycoside, and NSAID including COX-2 inhibitors, should be stopped for at least 2 days. Diabetic patients with preexisting renal impairment should withhold metformin for 48 hours because lactic acidosis may occur once CI-AKI develops. However, patients with normal renal function who are taking metformin are not at risk of CI-AKI and should be assessed according to their overall clinical conditions [20]. 
The pathogenic role of angiotensin-converting enzyme (ACE) inhibitors in CI-AKI is still controversial. Some studies pointed out that ACE inhibitors were effective in the prevention of CI-AKI, while some concluded that they were associated with increased risk of CI-AKI, especially for patients with preexisting renal impairment. On one hand, experimental data suggest that activated renin-angiotensinaldosterone system, enhanced endothelin-1, and increased ROS play important roles in the pathogenesis of CI-AKI and these can be inhibited by ACE inhibitors. On the other hand, ACE inhibitors impair angiotensin II synthesis. In the presence of contrast-induced acute reduction of renal blood flow, blunting the vasoconstriction of efferent arterioles by decreased angiotensin II levels may have a deleterious effect on GFR via decreasing the intraglomerular pressure. These opposite effects probably explain why the studies to date have provided inconclusive results on this issue [21].

Kwok et al. [22] performed systematic review and metaanalysis involving 9 different interventions for CI-AKI; furosemide was shown to increase the risk of CI-AKI (RR $3.27,95 \%$ CI 1.48-7.26). Therefore, this drug should be withheld for avoiding dehydration status before receiving contrast media. However, in patients who still had volume overload such as congestive heart failure or pulmonary edema, furosemide should be used to establish normovolemia prior to contrast exposure.

Lapi et al. [23] reported that current use of a double therapy combination of either diuretics or ACE inhibitors or angiotensin receptor blockers with NSAIDs was not associated with an increased rate of CI-AKI. On the contrary, the current use of a triple therapy combination was correlated with an augmented rate of CI-AKI (rate ratio 1.31, 95\% confidence interval 1.12 to 1.53 ). The highest risk was observed in the first 30 days of use (rate ratio 1.82, 95\% confidence interval 1.35 to 2.46 ).

3.3. Volume Expansion. All patients receiving contrast media should have an optimal volume status at the time of exposure. Indeed, volume supplementation plays an important role in the prevention of CI-AKI via two mechanisms. First, expansion of the intravascular space is thought to blunt the vasoconstrictive effect of contrast on the renal medulla via suppression of vasopressin secretion, inhibition of the reninangiotensin axis, and increased synthesis of vasodilatory renal prostaglandins. Second, intravenous fluid replacement is believed to attenuate the direct toxic effect of contrast agents on tubular epithelial cells by decreasing the concentration and viscosity of contrast media in the tubular lumen. This attenuating effect is the result of volume-mediated inhibition of proximal tubular salt and water reabsorption and decreasing contact time from the associated increase in tubular flow [24].

In patients without heart failure, parenteral isotonic normal saline $(0.9 \% \mathrm{NaCl})$ without any diuretics should be started 12 hours prior to contrast media administration with an infusion rate of $1 \mathrm{~mL} / \mathrm{kg} /$ hour and continued for 24 hours. The use of sodium bicarbonate $\left(\mathrm{NaHCO}_{3}\right)$ infusion may not only allow for shorter period of volume supplementation but also further reduce the generation of injurious ROS.
Typically, patients should receive $154 \mathrm{mEq} / \mathrm{L}$ of $\mathrm{NaHCO}_{3}$, as a bolus of $3 \mathrm{~mL} / \mathrm{kg} /$ hour for 1 hour prior to contrast media administration, followed by an infusion of $1 \mathrm{~mL} / \mathrm{kg} /$ hour for 6 hours after the procedure [25].

An earlier meta-analysis could not demonstrate the superior benefit of $\mathrm{NaHCO}_{3}$ when compared with normal saline [26]. Recent large meta-analysis studies revealed that $\mathrm{NaHCO}_{3}$ had a greater benefit than sodium chloride in terms of a change in serum creatinine but provided no significant differences in the occurrence of death and requirement for renal replacement therapy which were of much more important concerns [27-30]. Subgroup analysis by the type of contrast media was performed and suggested lower odds of CI-AKI with $\mathrm{NaHCO}_{3}$ in studies using LOCM (OR 0.40; 95\% CI $0.23-0.71, P=0.002$ ) compared with IOCM (OR 0.76; 95\% CI 0.41-1.43; $P=0.40$ ) [30]. The relatively low quality of the individual study, heterogeneity, and possible publication bias mean that only a limited recommendation can be made in favor of the use of $\mathrm{NaHCO}_{3}$ (Table 2).

Surprisingly, a recent meta-analysis [31] reported that the oral route of volume expansion may be as effective as the intravenous route for volume expansion for CIAKI prevention (odds ratio 1.19; 95\% CI 0.46, 3.10, $P=$ 0.73). In addition, Marenzi et al. [32] also demonstrated that furosemide-induced high urine output with matched hydration (receiving an initial $250 \mathrm{~mL}$ intravenous bolus of normal saline over $30 \mathrm{~min}$ followed by an intravenous bolus $0.5 \mathrm{mg} / \mathrm{kg}$ of furosemide) significantly reduced the risk of CI-AKI and might be associated with improved in-hospital outcome. Hydration infusion rate was automatically adjusted to precisely replace the patient's urine output. When a urine output rate $>300 \mathrm{~mL} /$ hour was obtained, patients underwent the coronary procedure. Matched fluid replacement was maintained during the procedure and for $4 \mathrm{~h}$ after treatment.

\subsection{Contrast Agent}

3.4.1. Type of Contrast Agent (Table 3). In a meta-analysis of 25 trials, the pooled odds ratio of CI-AKI with LOCM was 0.61 (95\% confidence interval (CI), 0.48-0.77) times that after high osmolar contrast media (HOCM). For CKD patients, this odds ratio was 0.5 (95\% CI, 0.36-0.68), while it was 0.75 (95\% CI, 0.52-1.1) in patients without prior renal insufficiency. These data suggest that HOCM is generally more nephrotoxic than LOCM and the use of LOCM may be beneficial, particularly in CKD patients [33]. Furthermore, a meta-analysis from 16 double-blind, randomized, controlled trials demonstrated significantly decreased risk of CI-AKI associated with the use of IOCM compared with LOCM, especially in patients with CKD or CKD and diabetes mellitus [34].

However, the risk of CI-AKI with two LOCM including iohexol and ioxaglate was significantly higher than other LOCM (for example, iopamidol, iopromide, and ioversol) and the IOCM (iodixanol) in many studies [35, 36]. In addition, iopamidol and iodixanol are preferable to the others because both reduce the risk of CI-AKI and are less costly and appear to be cost-effective when compared with iohexol or other LOCM [37]. 


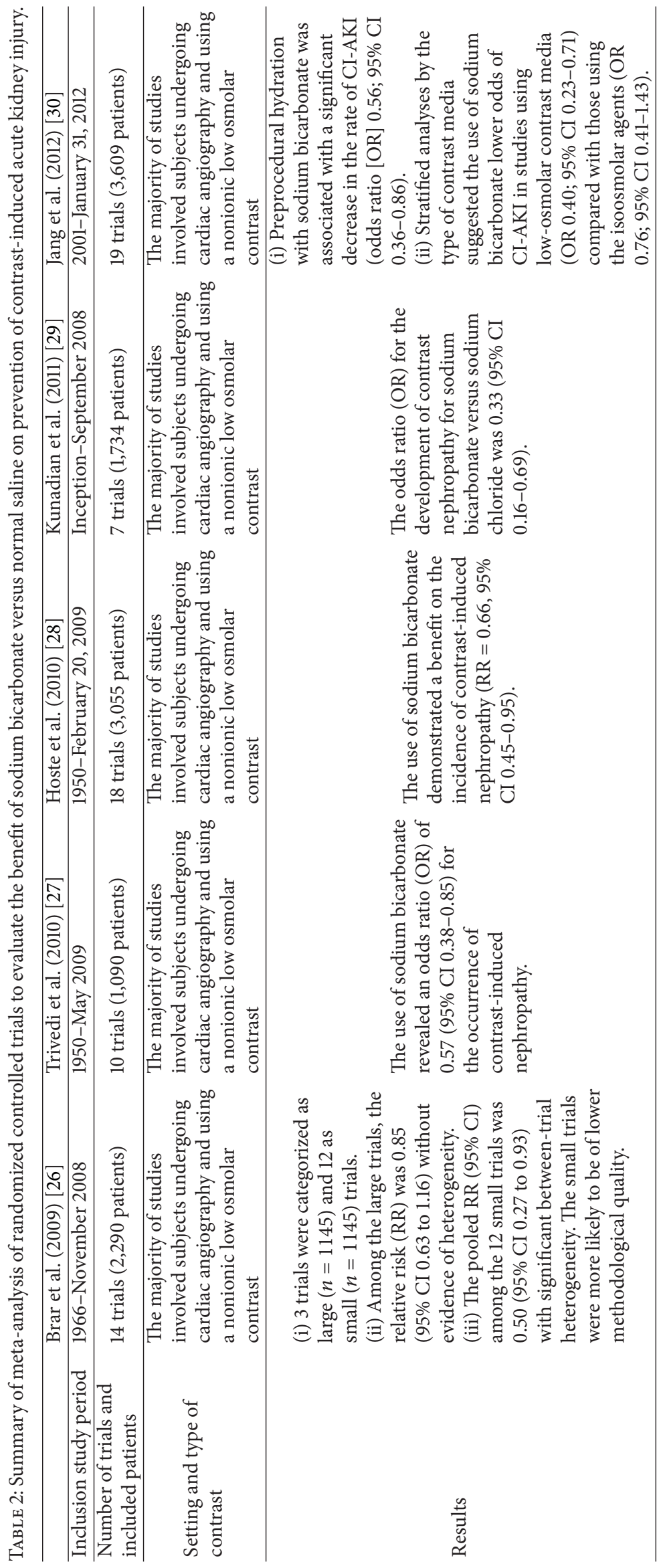


TABle 3: Type of contrast media.

\begin{tabular}{|c|c|c|c|c|c|c|}
\hline Type & Ionicity & Generic name & $\begin{array}{l}\text { Iodine content } \\
(\mathrm{mg} / \mathrm{mL})\end{array}$ & Osmolality & $\begin{array}{c}\text { Viscosity at } \\
20-25^{\circ} \mathrm{C} \\
(\mathrm{mPa} \cdot \mathrm{S})\end{array}$ & $\begin{array}{c}\text { Viscosity at } 37^{\circ} \mathrm{C} \\
(\mathrm{mPa} \cdot \mathrm{S})\end{array}$ \\
\hline HOCM & Ionic monomer & Diatrizoate & $300-370$ & $1500-2000$ & $3.3-16.4$ & $1.4-19.5$ \\
\hline HOCM & Ionic monomer & Metrizoate & $280-370$ & 2100 & $5-9$ & $2.8-5$ \\
\hline HOCM & Ionic monomer & Iothalamate & $141-480$ & $600-2400$ & $2-9$ & $1.5-5.0$ \\
\hline LOCM & Ionic dimer & Ioxaglate & $280-320$ & 600 & $12-15.7$ & $6-7.5$ \\
\hline LOCM & Nonionic monomer & Iohexol & $140-350$ & $322-844$ & $2.3-20.4$ & $1.5-10.4$ \\
\hline LOCM & Nonionic monomer & Iopamidol & $150-370$ & $300-832$ & $2.3-20.9$ & $1.5-9.5$ \\
\hline LOCM & Nonionic monomer & Iopromide & $150-400$ & $340-880$ & $2.3-22$ & $1.2-12.3$ \\
\hline LOCM & Nonionic monomer & Iopentol & $150-350$ & $310-810$ & $2.7-26.6$ & $1.7-12.0$ \\
\hline LOCM & Nonionic monomer & Iomeprol & $150-400$ & $301-730$ & $1.9-27.5$ & $1.4-12.6$ \\
\hline IOCM & Nonionic dimer & Iodixanol & $270-320$ & 290 & $12.7-26.6$ & $6.3-11.8$ \\
\hline IOCM & Nonionic dimer & Iotrolan & $240-300$ & $270-320$ & $6.8-16.4$ & $3.9-8.1$ \\
\hline
\end{tabular}

HOCM: high osmolar contrast media, LOCM: low-osmolar contrast media, and IOCM: isoosmolar contrast media.

Hence, the CI-AKI Consensus Working Panel suggests that, for intra-arterial administration in high-risk patients with CKD, particularly those with diabetes mellitus, nonionic IOCM are associated with the lowest risk of CI-AKI [38]. In addition, the current guidelines of the American College of Cardiology/American Heart Association recommend the use of either IOCM or LOCM other than iohexol and ioxaglate in CKD patients undergoing angiography [39]. Either IOCM or LOCM, except iohexol or ioxaglate, can be used in all patients. IOCM (iodixanol) may be a better choice for high-risk patients with $\mathrm{CKD}$ requiring intra-arterial administration.

3.4.2. Temperature. The contrast media should be prewarmed to $37^{\circ} \mathrm{C}$ for decreasing viscosity (Table 3 ).

3.4.3. Route of Administration. In a previous study, the incidences of AKI after intra-arterial and intravenous contrast media administrations were comparable [40]; however, IOCM (iodixanol) significantly decreased the risk of CI-AKI (risk ratio $(\mathrm{RR})=0.68 ; 95 \%$ CI $0.50-0.92 ; P=0.01$ ) with intra-arterial route, but not with intravenous application (RR $=0.75 ; 95 \%$ CI $0.44-1.26 ; P=0.27$ ), when compared with a pool of LOCM in a recent meta-analysis [41].

3.4.4. Contrast Volume and Multiple Studies. Multivariate analyses have established the correlation between higher dose of contrast media and risk for CI-AKI [42]. The CI-AKI Consensus Working Panel concludes that the higher contrast volumes $(>100 \mathrm{~mL})$ are associated with the higher rates of CIAKI in patients at risk [38].

Tan et al. [43] proposed the usage of the value derived from contrast media volume divided by creatinine clearance $(\mathrm{V} / \mathrm{CrCl})$ above 2.62 for predicting the risk for CI-AKI.

Automated Contrast Injector Systems (ACIS). Contrast volume is a major modifiable risk factor for CI-AKI. ACIS are believed to be associated with a reduction in the total volume of contrast media. Unfortunately, the use of ACIS was associated with a statistically significant lower in the average volume of contrast media with no difference in the incidence of CI-AKI or new need for dialysis [44].

In a recent meta-analysis in 79,694 patients from 10 studies, ACIS reduced contrast volume delivery by $45 \mathrm{~mL} /$ case $(P<0.001,95 \% \mathrm{CI}-54$ to -35$)$. The CI-AKI incidence was significantly reduced by $15 \%$ with an odds ratio of 0.85 $(P<0.001,95 \%$ CI 0.78 to 0.93$)$ for those using ACIS compared with manual injection [45]. In addition, a significant association between contrast media dose increment and high prevalence of CI-AKI was demonstrated in a recent cohort [46]. Therefore, using the lowest dose of contrast media should be emphasized.

In terms of the time interval between procedures that require intravascular contrast media administration, the Contrast Media Safety Committee of ESUR recommends that the ideally optimal time interval should be more than 2 weeks which are expected for recovery time of the kidney after acute injury from contrast media. When this is not possible, the interval should be as long as clinically acceptable.

In conclusion, the contrast media should be injected at the lowest possible dose. Repeated injection especially within 72 hours should be avoided and may be requested after 2-week period from the first exposure.

3.5. Follow-Up Assessment of Kidney Function. Serum creatinine at 48-72 hours following contrast media exposure should be assessed for CI-AKI detection.

3.6. Dialysis (Hemodialysis, Hemofiltration, or Peritoneal Dialysis). Contrast media are excreted mainly by glomerular filtration. Thus, there is a significant correlation between renal clearances of contrast media and glomerular filtration rate. Thus, renal excretion of contrast media will be delayed in CKD patients. A single session of hemodialysis (HD) can effectively remove 60-90\% of contrast media from the blood. Because most contrast media are middle-sized molecules, the main factors potentially affecting CI-AKI depend on HD efficacy of contrast media removal. Blood flow, membrane surface area, molecular size, transmembrane pressure, and 
dialysis time are important factors that contribute to the efficacy of HD [47]. Generally, several hemodialysis sessions are needed to eliminate all contrast media. Some studies have explored the necessity of immediate HD after intravascular injection of contrast media in chronic HD patients; the authors demonstrated no effective evidence for preventing CI-AKI [48]. The reasons why HD treatment was not beneficial in these studies are still unestablished. The rapid onset of renal injury after administration of contrast media might partly explain such finding. However, Marenzi et al. [49] reported the more efficacy of periprocedural hemofiltration in preventing $\mathrm{CI}-\mathrm{AKI}$ in $\mathrm{CKD}$ patients undergoing coronary interventions.

Peritoneal dialysis is also effective in removing contrast agents from the body but takes longer duration time period than HD. Three weeks of continuous ambulatory peritoneal dialysis is needed for completely removing the agent. Contrast media can be removed effectively by various peritoneal dialysis modalities, including intermittent peritoneal dialysis, automated peritoneal dialysis, and continuous ambulatory peritoneal dialysis [47].

A previous meta-analysis [50] and a recent meta-analysis [51] could not demonstrate the benefit of dialysis on the incidence of CI-AKI when compared with routine preventive care. However, in sensitivity analyses, limiting to only HD studies which could significantly reduce heterogeneity among the included studies, HD appeared to increase CI-AKI risk (RR 1.61; 95\% CI, 1.13-2.28) but had no effect on the need for permanent RRT or progression to end-stage renal disease (RR 1.47; 95\% CI, 0.56-3.89) [51] (Table 4).

Interestingly, Lee et al. [52] demonstrated the benefit of $\mathrm{HD}$ after coronary angiogram in patients with CKD stage $\mathrm{V}$ with a mean baseline creatinine clearance of $12.9 \mathrm{~mL} / \mathrm{min} /$ $1.73 \mathrm{~m}^{2}$. Approximately, $60 \%$ had diabetes mellitus and the mean volume of total contrast media (iohexol) was more than $100 \mathrm{~mL}$ that contributed to a high risk of CI-AKI. All patients were given intravenous normal saline at $1 \mathrm{~mL} / \mathrm{kg} /$ hour for 6 hours before and 12 hours after contrast media exposure. HD was prescribed by using a high-flux dialyzer. The blood flow was $150 \mathrm{~mL} / \mathrm{min}$, the duration of dialysis was 4 hours, and the dialysate flow was $500 \mathrm{~mL} / \mathrm{min}$. To lessen the hemodynamic changes, $200 \mathrm{~mL}$ normal saline priming was administered before dialysis and no fluid removal was prescribed in the dialysis group. HD was initiated at an interval of $81 \pm 32 \mathrm{~min}$, ranging from 45 to $180 \mathrm{~min}$, after exposure to the contrast media. A potential important limitation of this study is the use of serum creatinine to diagnose CI-AKI. Indeed, any initial reduction of serum creatinine in HD group is likely a falsely beneficial effect resulting from creatinine removal. The bicarbonate dialysate used and additional fluid replacement might be another important effect. However, the prophylactic dialysis seems not to be important even in advanced CKD.

In addition, the risks of dialysis procedures and the much greater cost should be considered. As such, the Contrast Media Safety Committee of ESUR states that there is no need to schedule the dialysis in relation to the injection of contrast media or the injection of contrast agent in relation to the dialysis program. Hemodialysis does not protect the poorly functioning kidney against CI-AKI [53].
3.7. Coronary Sinus Contrast Removal System. As a strong relationship between contrast load and incidence of CIN is obviously demonstrated, alternative strategies to limit the systemic contrast exposure are being developed. The CINCOR catheter (Contrast Removal System, Osprey Medical, St. Paul, Minnesota, USA) has been innovated for removing contrast media from coronary sinus shortly after contrast delivery [54]. Contrast media were effectively withdrawn (44 $\pm 8 \%)$ as assessed by fluoroscopy [55]. Early data demonstrated the benefit of the procedure in attenuating CI-AKI compared with the standard care in small cohort studies $[56,57]$. However, one limitation of this system was the requirement for 14 French internal jugular vein sheath insertion. A largescale randomized trial to evaluate the capacity of this device to reduce the risk of CI-AKI and its complications is required.

3.8. Oxygenation Support. Sekiguchi et al. [58] randomly allocated 349 eligible patients who underwent elective coronary angiography and/or percutaneous coronary intervention to either an oxygenation group (oxygen administration via nasal cannula; $2 \mathrm{~L} / \mathrm{min}$ of oxygen from $10 \mathrm{~min}$ before the procedure to the end of the procedure; $n=174$ ) or to a control group (room air; $n=175$ ). Continuous infusion of isotonic saline solution $(1 \mathrm{~mL} / \mathrm{kg} /$ hour $)$ was administered 12 hours before the procedure until 12 hours after the procedure in both groups. The $\mathrm{PaO}_{2}$ at the baseline was significantly higher in the oxygen preconditioning group than the control group $(134 \pm 28$ versus $90 \pm 12 \mathrm{~mm} \mathrm{Hg}, P<0.001)$. The authors demonstrated that the oxygen preconditioning reduced the incidence of CI-AKI, particularly in CKD patients, via decreased intrarenal hypoxia. Therefore, this simple strategy to attenuate CI-AKI might be beneficial in CKD patients.

3.9. Remote Ischemic Preconditioning (IPC). Er et al. [59] demonstrated that remote IPC induced by intermittent upper-arm ischemia prior to elective coronary angiography dramatically reduced the incidence of CI-AKI in patients with CKD and those at high risk of CI-AKI (OR 0.21, 95\% CI $0.07-0.57, P=0.002)$. The IPC was performed as 4 cycles of alternating 5-minute inflation and 5-minute deflation of a standard upper-arm blood pressure cuff to the individual's systolic blood pressure plus $50 \mathrm{~mm} \mathrm{Hg}$ to induce transient and repetitive arm ischemia and reperfusion. Although the protective mechanism of IPC is still unestablished, it is postulated that a remote organ might release humoral factors such as adenosine, bradykinin, or erythropoietin into the systemic circulation, all of which subsequently protect the remote region or organ.

\section{Conclusion}

The Contrast Media Safety Committee of ESUR [18] has updated its guidelines on CI-AKI (Table 5). First, identify high-risk patients, especially those with eGFR < $60 \mathrm{~mL} / \mathrm{min} / 1.73 \mathrm{~m}^{2}$, diabetes mellitus, recent nephrotoxic exposure, and intra-arterial route. In at-risk patients, consider an alternative imaging method, start volume expansion, and utilize the lowest dose of contrast media consistent with 


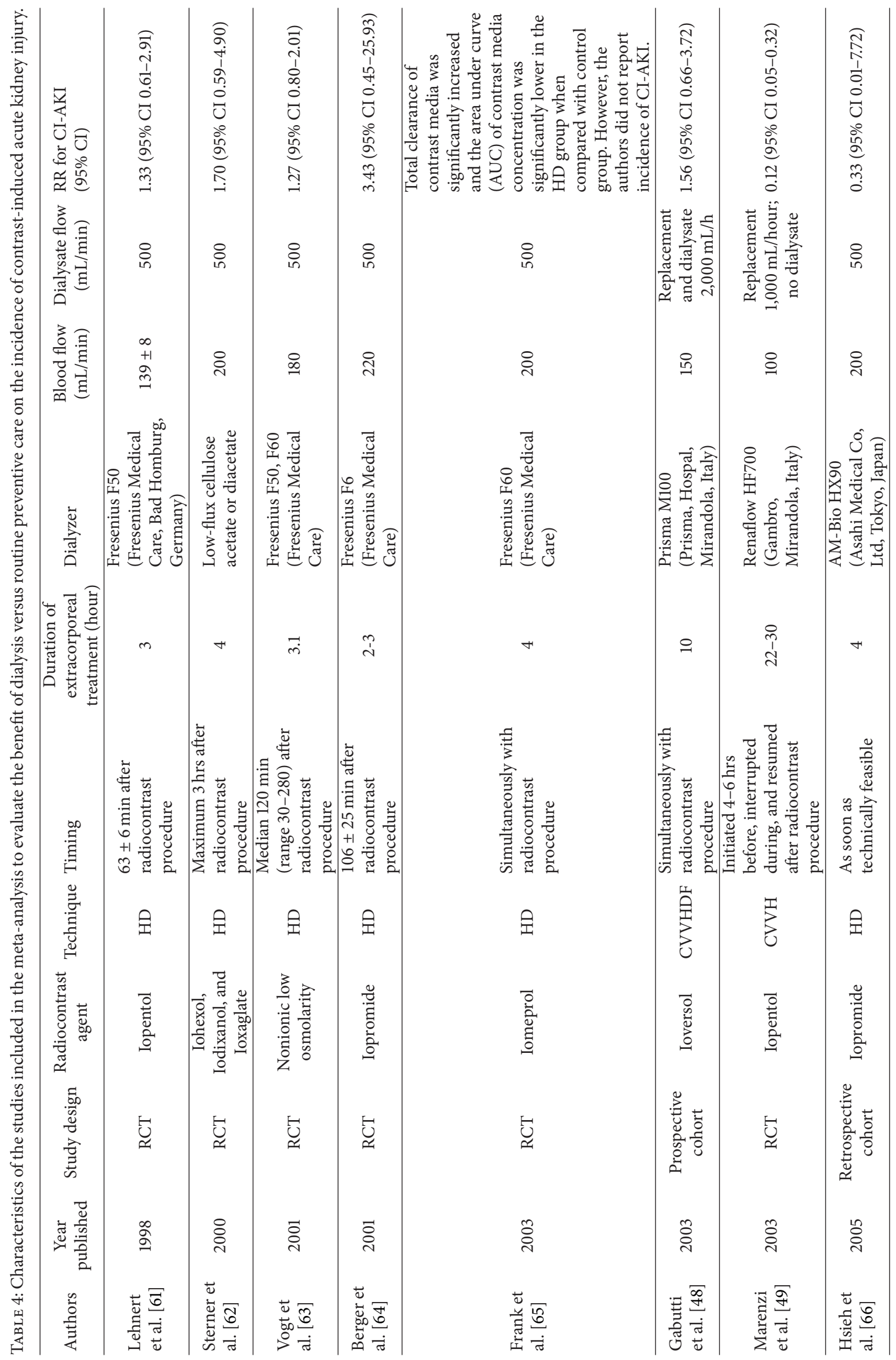




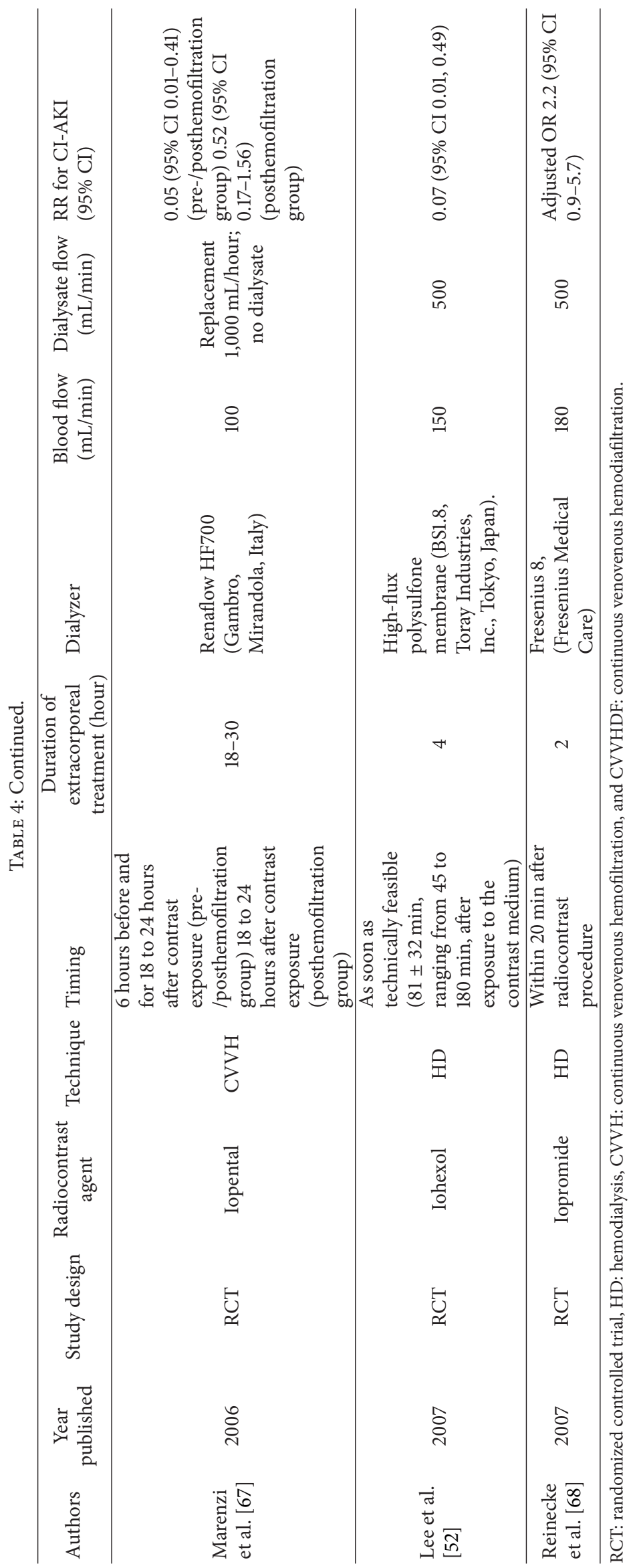




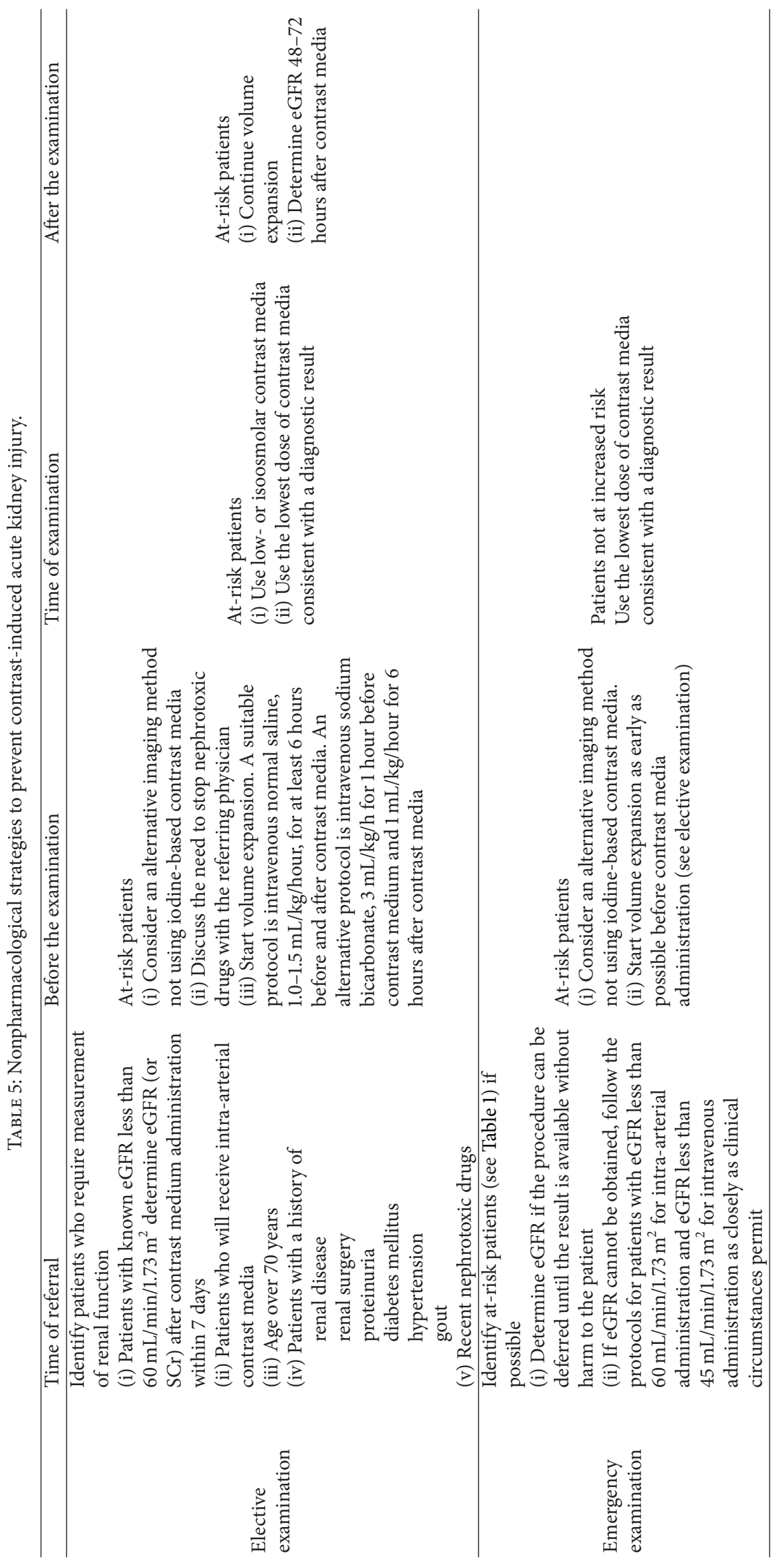


a diagnostic result. Finally, determining eGFR 48-72 hours after receiving contrast media should be performed for CIAKI detection.

Similarly, the KDIGO Clinical Practice Guidelines on Acute Kidney Injury [60] recommend that balancing the risk for CI-AKI against the benefit of administering contrast media should be firstly considered. Alternative imaging methods not requiring contrast media administration in patients at increased risk for CI-AKI so long as these yield the same diagnostic accuracy might be required. Before an intervention which encompasses a risk for CI-AKI, a baseline serum creatinine should be determined. Volume expansion with either isotonic sodium chloride or sodium bicarbonate solutions, rather than no volume expansion in patients at increased risk for CI-AKI, should be considered during hospitalization. In high-risk patients, a repeated serum creatinine should be performed at 12 and 72 hours after administration of contrast media. Prophylactic intermittent hemodialysis or hemofiltration did not have strong evidence in updated data for the purpose of CI-AKI prevention only.

In conclusion, contrast-induced AKI (CI-AKI) has been one of the leading causes for hospital-acquired AKI and is associated with independent risk for adverse clinical outcomes including morbidity and mortality. To prevent CI-AKI in patients who are receiving contrast media, every effort is required, including routine identification of at-risk patients, the use of appropriate hydration regimens, withdrawal of nephrotoxic drugs, selection of LOCM or IOCM, and using the minimum volume of contrast media as possible. There is no need to schedule the dialysis in relation to the injection of contrast media or the injection of contrast agent in relation to the dialysis program. Hemodialysis does not protect the poorly functioning kidney against CI-AKI.

\section{Conflict of Interests}

The authors declare that there is no conflict of interests regarding the publication of this paper.

\section{References}

[1] P. Susantitaphong, D. N. Cruz, J. Cerda et al., "World incidence of AKI: a meta-analysis," Clinical Journal of the American Society of Nephrology, vol. 8, no. 9, pp. 1482-1493, 2013.

[2] H. S. Thomsen, S. K. Morcos, and B. J. Barrett, "Contrastinduced nephropathy: the wheel has turned 360 degrees," Acta Radiologica, vol. 49, no. 6, pp. 646-657, 2008.

[3] J. Kooiman, S. M. Pasha, W. Zondag et al., "Meta-analysis: serum creatinine changes following contrast enhanced CT imaging," European Journal of Radiology, vol. 81, no. 10, pp. 2554-2561, 2012.

[4] M. T. James, S. M. Samuel, M. A. Manning et al., "Contrastinduced acute kidney injury and risk of adverse clinical outcomes after coronary angiography: a systematic review and meta-analysis," Circulation: Cardiovascular Interventions, vol. 6, no. 1, pp. 37-43, 2013.

[5] R. W. Geenen, H. J. Kingma, and A. J. van der Molen, “Contrastinduced nephropathy: pharmacology, pathophysiology and prevention," Insights into Imaging, vol. 4, no. 6, pp. 811-820, 2013.
[6] P. C. Wong, Z. Li, J. Guo, and A. Zhang, "Pathophysiology of contrast-induced nephropathy," International Journal of Cardiology, vol. 158, no. 2, pp. 186-192, 2012.

[7] C.-F. Chang and C.-C. Lin, "Current concepts of contrastinduced nephropathy: a brief review," Journal of the Chinese Medical Association, vol. 76, no. 12, pp. 673-681, 2013.

[8] J. Tumlin, F. Stacul, A. Adam et al., "Pathophysiology of contrast-induced nephropathy," American Journal of Cardiology, vol. 98, no. 6, supplement 1, pp. 14-20, 2006.

[9] S. I. Moos, D. N. H. van Vemde, J. Stoker, and S. Bipat, "Contrast induced nephropathy in patients undergoing intravenous (IV) contrast enhanced computed tomography (CECT) and the relationship with risk factors: a meta-analysis," European Journal of Radiology, vol. 82, no. 9, pp. e387-e399, 2013.

[10] D. Sany, H. Refaat, Y. Elshahawy, A. Mohab, and H. Ezzat, "Frequency and risk factors of contrast-induced nephropathy after cardiac catheterization in type II diabetic patients: a study among Egyptian patients," Renal Failure, vol. 36, no. 2, pp. 191197, 2014.

[11] M. Saritemur, M. Turkeli, K. Kalkan, I. H. Tanboga, and E. Aksakal, "Relation of uric acid and contrast-induced nephropathy in patients undergoing primary percutaneous coronary intervention in the ED," The American Journal of Emergency Medicine, vol. 32, no. 2, pp. 119-123, 2014.

[12] A. Kaya, Y. Kaya, S. Topçu et al., "Neutrophil-to-lymphocyte ratio predicts contrast-induced nephropathy in patients undergoing primary percutaneous coronary intervention," Angiology, vol. 65, no. 1, pp. 51-56, 2014.

[13] S.-S. Huang, P.-H. Huang, H.-B. Leu, T. -C. Wu, S. -J. Lin, and J.-W. Chen, "Association of central pulse pressure with contrastinduced nephropathy and clinical outcomes in patients undergoing coronary intervention," Journal of Hypertension, vol. 31, no. 11, pp. 2187-2194, 2013.

[14] H. Ucar, M. Gur, A. Yildirim et al., "Increased aortic stiffness predicts contrast-induced nephropathy in patients with stable coronary artery disease undergoing percutaneous coronary intervention," Angiology, 2013.

[15] W. H. Li, D. Y. Li, F. Han, T.-D. Xu, Y.-B. Zhang, and H. Zhu, "Impact of anemia on contrast-induced nephropathy (CIN) in patients undergoing percutaneous coronary interventions," International Urology and Nephrology, vol. 45, no. 4, pp. 10651070, 2013.

[16] R. Murakami, S.-I. Kumita, H. Hayashi et al., "Anemia and the risk of contrast-induced nephropathy in patients with renal insufficiency undergoing contrast-enhanced MDCT," European Journal of Radiology, vol. 82, no. 10, pp. e521-e524, 2013.

[17] H. S. Gurm, M. Seth, J. Kooiman, and D. Share, "A novel tool for reliable and accurate prediction of renal complications in patients undergoing percutaneous coronary intervention," Journal of the American College of Cardiology, vol. 61, no. 22, pp. 2242-2248, 2013.

[18] F. Stacul, A. J. van der Molen, P. Reimer et al., "Contrast induced nephropathy: updated ESUR Contrast Media Safety Committee guidelines," European Radiology, vol. 21, no. 12, pp. 2527-2541, 2011.

[19] A. J. Cho, J. E. Lee, J. Y. Yoon et al., "Effect of an electronic alert on risk of contrast-induced acute kidney injury in hospitalized patients undergoing computed tomography," American Journal of Kidney Diseases, vol. 60, no. 1, pp. 74-81, 2012.

[20] S. Nawaz, T. Cleveland, P. A. Gaines, and P. Chan, "Clinical risk associated with contrast angiography in metformin treated 
patients: a clinical review," Clinical Radiology, vol. 53, no. 5, pp. 342-344, 1998.

[21] X. Li, T. Li, and H. Cong, "Is angiotensin-converting enzyme inhibitor a contraindication for contrast-induced nephropathy prophylaxis? A review about its paradox," Cardiovascular Therapeutics, vol. 30, no. 5, pp. 273-276, 2012.

[22] C. S. Kwok, C. L. Pang, J. K. Yeong, and Y. K. Loke, "Measures used to treat contrast-induced nephropathy: overview of reviews," The British Journal of Radiology, vol. 86, no. 1021, Article ID 20120272, 2013.

[23] F. Lapi, L. Azoulay, H. Yin, S. J. Nessim, and S. Suissa, “Concurrent use of diuretics, angiotensin converting enzyme inhibitors, and angiotensin receptor blockers with non-steroidal antiinflammatory drugs and risk of acute kidney injury: nested case-control study," BMJ, vol. 346, Article ID e8525, 2013.

[24] S. D. Weisbord and P. M. Palevsky, "Prevention of contrastinduced nephropathy with volume expansion," Clinical Journal of the American Society of Nephrology, vol. 3, no. 1, pp. 273-280, 2008.

[25] G. J. Merten, W. P. Burgess, L. V. Gray et al., "Prevention of contrast-induced nephropathy with sodium bicarbonate: a randomized controlled trial," Journal of the American Medical Association, vol. 291, no. 19, pp. 2328-2334, 2004.

[26] S. S. Brar, S. Hiremath, G. Dangas, R. Mehran, S. K. Brar, and M. B. Leon, "Sodium bicarbonate for the prevention of contrast induced-acute kidney injury: a systematic review and metaanalysis," Clinical Journal of the American Society of Nephrology, vol. 4, no. 10, pp. 1584-1592, 2009.

[27] H. Trivedi, R. Nadella, and A. Szabo, "Hydration with sodium bicarbonate for the prevention of contrast-induced nephropathy: a meta-analysis of randomized controlled trials," Clinical Nephrology, vol. 74, no. 4, pp. 288-296, 2010.

[28] E. A. Hoste, J. J. de Waele, S. A. Gevaert, S. Uchino, and J. A. Kellum, "Sodium bicarbonate for prevention of contrastinduced acute kidney injury: a systematic review and metaanalysis," Nephrology Dialysis Transplantation, vol. 25, no. 3, pp. 747-758, 2010.

[29] V. Kunadian, A. Zaman, I. Spyridopoulos, and W. Qiu, "Sodium bicarbonate for the prevention of contrast induced nephropathy: a meta-analysis of published clinical trials," European Journal of Radiology, vol. 79, no. 1, pp. 48-55, 2011.

[30] J.-S. Jang, H.-Y. Jin, J.-S. Seo et al., "Sodium bicarbonate therapy for the prevention of contrast-induced acute kidney injury-a systematic review and meta-analysis," Circulation Journal, vol. 76, no. 9, pp. 2255-2265, 2012.

[31] S. Hiremath, A. Akbari, W. Shabana, D. A. Fergusson, and G. A. Knoll, "Prevention of contrast-induced acute kidney injury: is simple oral hydration similar to intravenous? A systematic review of the evidence," PLoS ONE, vol. 8, no. 3, Article ID e60009, 2013.

[32] G. Marenzi, C. Ferrari, I. Marana et al., "Prevention of contrast nephropathy by furosemide with matched hydration: the MYTHOS (induced diuresis with matched hydration compared to standard hydration for contrast induced nephropathy prevention) trial," JACC: Cardiovascular Interventions, vol. 5, no. 1, pp. 90-97, 2012.

[33] B. J. Barrett and E. J. Carlisle, "Metaanalysis of the relative nephrotoxicity of high- and low-osmolality iodinated contrast media," Radiology, vol. 188, no. 1, pp. 171-178, 1993.

[34] P. A. McCullough, M. E. Bertrand, J. A. Brinker, and F. Stacul, "A meta-analysis of the renal safety of isosmolar iodixanol compared with low-osmolar contrast media," Journal of the American College of Cardiology, vol. 48, no. 4, pp. 692-699, 2006.

[35] R. Solomon, "The role of osmolality in the incidence of contrastinduced nephropathy: a systematic review of angiographic contrast media in high risk patients," Kidney International, vol. 68, no. 5, pp. 2256-2263, 2005.

[36] M. Reed, P. Meier, U. U. Tamhane, K. B. Welch, M. Moscucci, and H. S. Gurm, "The relative renal safety of iodixanol compared with low-osmolar contrast media: a meta-analysis of randomized controlled trials," JACC: Cardiovascular Interventions, vol. 2, no. 7, pp. 645-654, 2009.

[37] L. A. Chicaiza-Becerra, M. García-Molina, and O. Gamboa, "Cost-effectiveness of iso- versus low-osmolality contrast media in outpatients with high risk of contrast medium-induced nephropathy," Biomedica, vol. 32, no. 2, pp. 182-188, 2012.

[38] C. Davidson, F. Stacul, P. A. McCullough et al., "Contrast medium use," American Journal of Cardiology, vol. 98, no. 6, supplement 1, pp. 42-58, 2006.

[39] F. G. Kushner, M. Hand, S. C. Smith Jr. et al., "2009 focused updates: ACC/AHA guidelines for the management of patients with ST-elevation myocardial infarction (updating the 2004 guideline and 2007 focused update) and ACC/AHA/SCAI guidelines on percutaneous coronary intervention (updating the 2005 guideline and 2007 focused update)," Journal of the American College of Cardiology, vol. 54, no. 23, pp. 2205-2241, 2009.

[40] J. Kooiman, P. A. Le Haen, G. Gezgin et al., "Contrastinduced acute kidney injury and clinical outcomes after intraarterial and intravenous contrast administration: risk comparison adjusted for patient characteristics by design," American Heart Journal, vol. 165, no. 5, pp. 793.e1-799.e1, 2013.

[41] M. Dong, Z. Jiao, T. Liu, F. Guo, and G. Li, "Effect of administration route on the renal safety of contrast agents: a meta-analysis of randomized controlled trials," Journal of Nephrology, vol. 25, no. 3, pp. 290-301, 2012.

[42] E. Nikolsky, R. Mehran, D. Turcot et al., "Impact of chronic kidney disease on prognosis of patients with diabetes mellitus treated with percutaneous coronary intervention," American Journal of Cardiology, vol. 94, no. 3, pp. 300-305, 2004.

[43] N. Tan, Y. Liu, J. Y. Chen et al., "Use of the contrast volume or grams of iodine-to-creatinine clearance ratio to predict mortality after percutaneous coronary intervention," American Heart Journal, vol. 165, no. 4, pp. 600-608, 2013.

[44] H. S. Gurm, D. Smith, D. Share et al., "Impact of automated contrast injector systems on contrast use and contrast-associated complications in patients undergoing percutaneous coronary interventions," JACC: Cardiovascular Interventions, vol. 6, no. 4, pp. 399-405, 2013.

[45] K. D. Minsinger, H. M. Kassis, C. A. Block, M. Sidhu, and J. R. Brown, "Meta-analysis of the effect of automated contrast injection devices versus manual injection and contrast volume on risk of contrast-induced nephropathy," The American Journal of Cardiology, vol. 113, no. 1, pp. 49-53, 2013.

[46] M. Yamamoto, K. Hayashida, G. Mouillet et al., "Renal function-based contrast dosing predicts acute kidney injury following transcatheter aortic valve implantation," JACC: Cardiovascular Interventions, vol. 6, no. 5, pp. 479-486, 2013.

[47] G. Deray, "Dialysis and iodinated contrast media," Kidney international, vol. 69, no. 100, pp. S25-S29, 2006.

[48] L. Gabutti, C. Marone, M. Monti et al., "Does continuous venovenous hemodiafiltration concomitant with radiological 
procedures provide a significant and safe removal of the iodinated contrast ioversol?" Blood Purification, vol. 21, no. 2, pp. 152-157, 2003.

[49] G. Marenzi, I. Marana, G. Lauri et al., "The prevention of radiocontrast-agent-induced nephropathy by hemofiltration," The New England Journal of Medicine, vol. 349, no. 14, pp. 13331340, 2003.

[50] D. N. Cruz, M. A. Perazella, R. Bellomo et al., "Extracorporeal blood purification therapies for prevention of radiocontrastinduced nephropathy: a systematic review," American Journal of Kidney Diseases, vol. 48, no. 3, pp. 361-371, 2006.

[51] D. N. Cruz, C. Y. Goh, G. Marenzi, V. Corradi, C. Ronco, and M. A. Perazella, "Renal replacement therapies for prevention of radiocontrast-induced nephropathy: a systematic review," The American Journal of Medicine, vol. 125, no. 1, pp. 66.e3-78.e3, 2012.

[52] P.-T. Lee, K.-J. Chou, C.-P. Liu et al., "Renal protection for coronary angiography in advanced renal failure patients by prophylactic hemodialysis. A randomized controlled trial," Journal of the American College of Cardiology, vol. 50, no. 11, pp. 1015-1020, 2007.

[53] S. K. Morcos, H. S. Thomsen, J. A. Webb et al., "Dialysis and contrast media," European Radiology, vol. 12, no. 12, pp. 3026-3030, 2002.

[54] I. Michishita and Z. Fujii, "A novel contrast removal system from the coronary sinus using an adsorbing column during coronary angiography in a porcine model," Journal of the American College of Cardiology, vol. 47, no. 9, pp. 1866-1870, 2006.

[55] H. D. Danenberg, C. Lotan, B. Varshitski, S. Rosenheck, and A. T. Weiss, "Removal of contrast medium from the coronary sinus during coronary angiography: feasibility of a simple and available technique for the prevention of nephropathy," Cardiovascular Revascularization Medicine, vol. 9, no. 1, pp. 9-13, 2008.

[56] S. J. Duffy, P. Ruygrok, C. P. Juergens et al., "Removal of contrast media from the coronary sinus attenuates renal injury after coronary angiography and intervention," Journal of the American College of Cardiology, vol. 56, no. 6, pp. 525-526, 2010.

[57] T. Watson, J. S. Burd, and P. N. Ruygrok, "Prevention of contrast induced nephropathy during coronary angiography with a coronary sinus contrast removal system sited from the femoral vein," International Journal of Cardiology, vol. 165, no. 1, pp. e9e10, 2013.

[58] H. Sekiguchi, Y. Ajiro, Y. Uchida et al., "Oxygen pre-conditioning prevents contrast-induced nephropathy (OPtion CIN Study)," Journal of the American College of Cardiology, vol. 62, no. 2, pp. 162-163, 2013.

[59] F. Er, A. M. Nia, H. Dopp et al., "Ischemic preconditioning for prevention of contrast medium-induced nephropathy: randomized pilot RenPro Trial (Renal Protection Trial)," Circulation, vol. 126, no. 3, pp. 296-303, 2012.

[60] Improving Global Outcomes (KDIGO) Acute Kidney Injury Work Group, "KDIGO clinical practice guideline for acute kidney injury," Kidney International Supplements, vol. 2, no. 1, pp. 1-138, 2012.

[61] T. Lehnert, E. Keller, K. Gondolf, T. Schäffner, H. Pavenstädt, and P. Schollmeyer, "Effect of haemodialysis after contrast medium administration in patients with renal insufficiency," Nephrology Dialysis Transplantation, vol. 13, no. 2, pp. 358-362, 1998.

[62] G. Sterner, B. Frennby, J. Kurkus, and U. Nyman, "Does postangiographic hemodialysis reduce the risk of contrast-medium nephropathy?" Scandinavian Journal of Urology and Nephrology, vol. 34, no. 5, pp. 323-326, 2000.
[63] B. Vogt, P. Ferrari, C. Schönholzer et al., "Prophylactic hemodialysis after radiocontrast media in patients with renal insufficiency is potentially harmful," The American Journal of Medicine, vol. 111, no. 9, pp. 692-698, 2001.

[64] E. D. Berger, B. D. Bader, J. Bösker, T. Risler, and C. M. Erley, "Contrast media-induced kidney failure cannot be prevented by hemodialysis," Deutsche Medizinische Wochenschrift, vol.126, no. 7, pp. 162-166, 2001.

[65] H. Frank, D. Werner, V. Lorusso et al., "Simultaneous hemodialysis during coronary angiography fails to prevent radiocontrastinduced nephropathy in chronic renal failure," Clinical Nephrology, vol. 60, no. 3, pp. 176-182, 2003.

[66] Y.-C. Hsieh, C.-T. Ting, T.-J. Liu, C.-L. Wang, Y.-T. Chen, and W.-L. Lee, "Short- and long-term renal outcomes of immediate prophylactic hemodialysis after cardiovascular catheterizations in patients with severe renal insufficiency," International Journal of Cardiology, vol. 101, no. 3, pp. 407-413, 2005.

[67] G. Marenzi, G. Lauri, J. Campodonico et al., "Comparison of two hemofiltration protocols for prevention of contrastinduced nephropathy in high-risk patients," The American Journal of Medicine, vol. 119, no. 2, pp. 155-162, 2006.

[68] H. Reinecke, M. Fobker, J. Wellmann et al., "A randomized controlled trial comparing hydration therapy to additional hemodialysis or $\mathrm{N}$-acetylcysteine for the prevention of contrast medium-induced nephropathy: the Dialysis-versus-Diuresis (DVD) trial," Clinical Research in Cardiology, vol. 96, no. 3, pp. 130-139, 2007. 


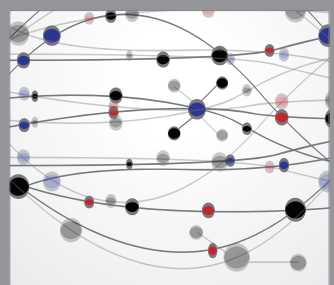

The Scientific World Journal
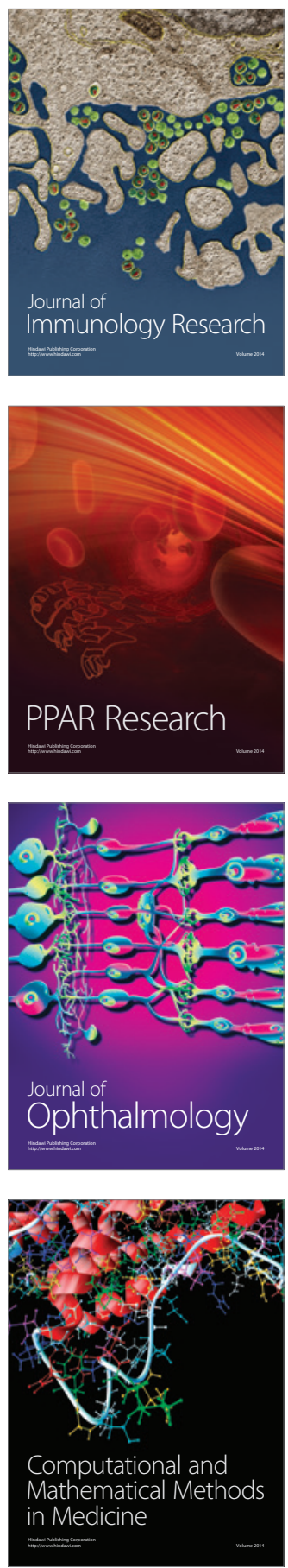

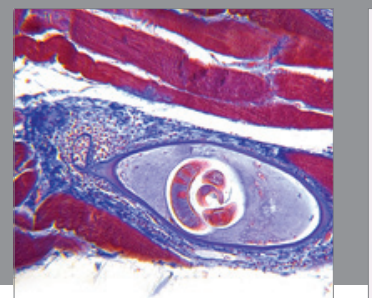

Gastroenterology

Research and Practice
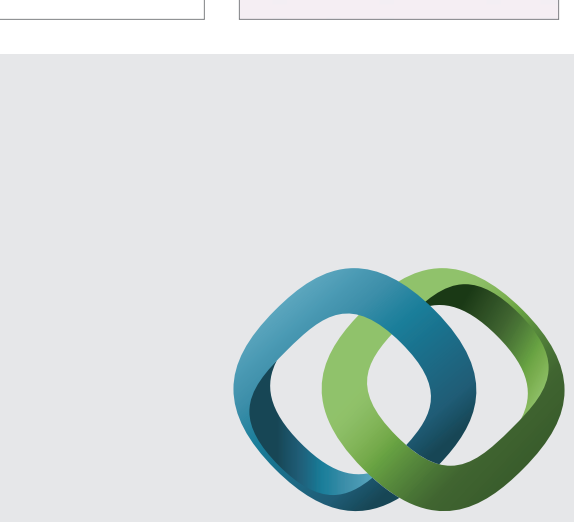

\section{Hindawi}

Submit your manuscripts at

http://www.hindawi.com
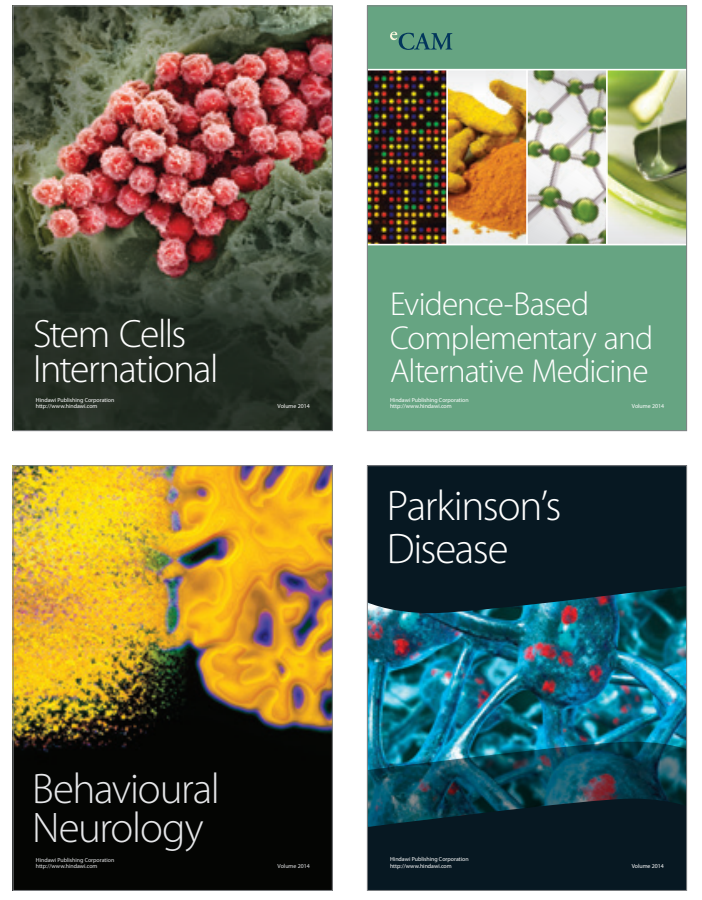
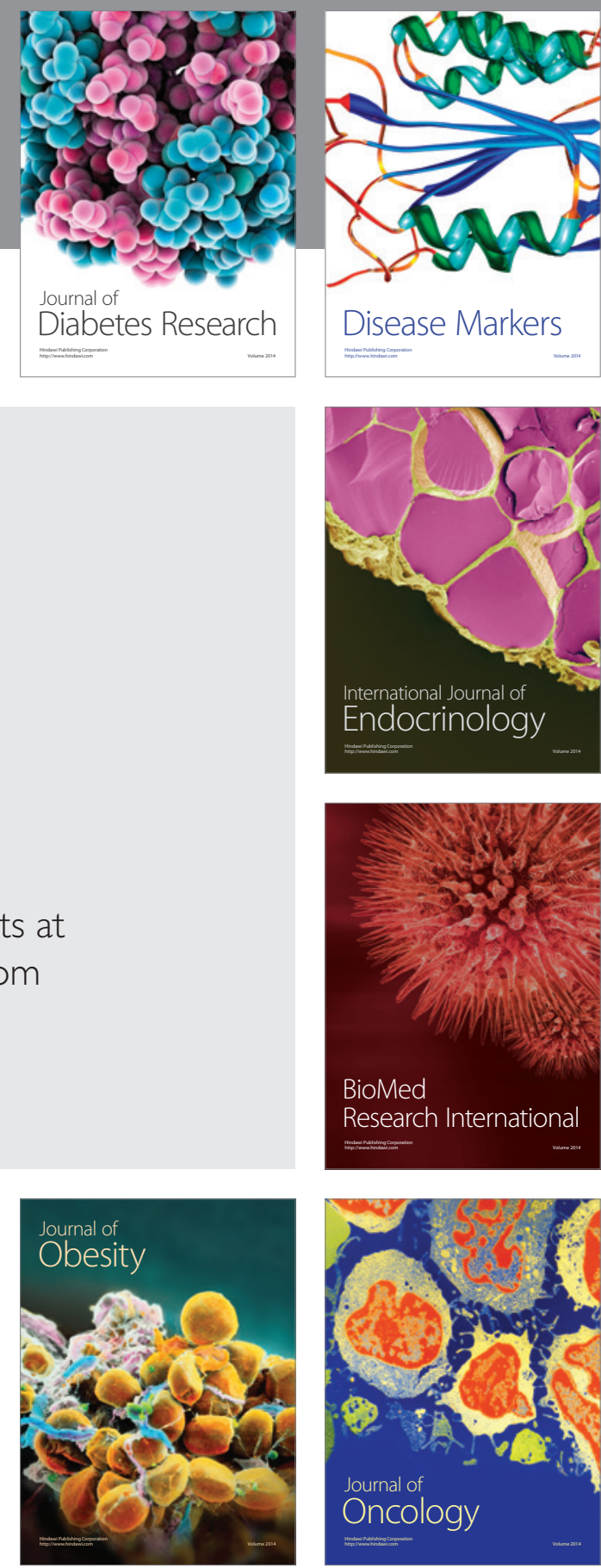

Disease Markers
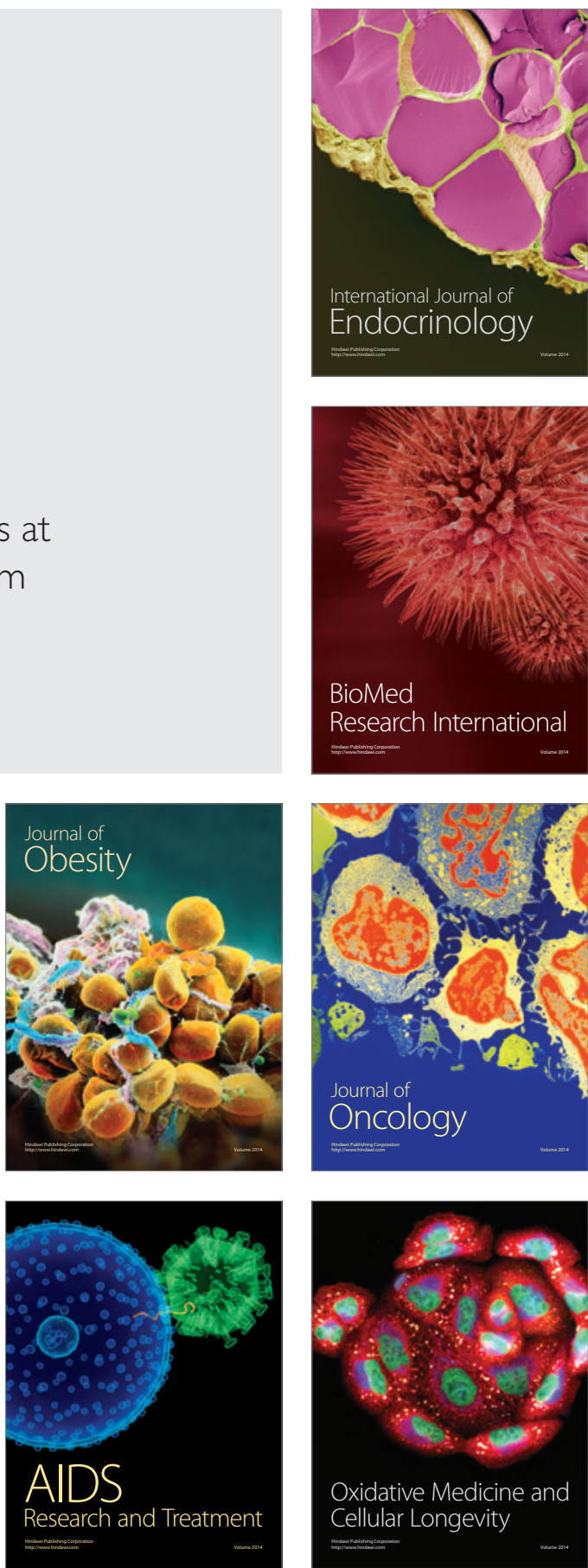